\title{
Primary Renal Carcinoid: Two Rare Cases at a Single Center
}

\author{
Emily F. Kelly ${ }^{1}$, Zachary M. Connelly ${ }^{1}$, Mackenzie J. Noonan ${ }^{1}$, Xin Gu ${ }^{2}$, Nazih Khater ${ }^{1}$ \\ 1. Urology, Louisiana State University Health Shreveport, Shreveport, USA 2. Pathology, Louisiana State University \\ Health Shreveport, Shreveport, USA
}

Corresponding author: Nazih Khater, nkhate@lsuhsc.edu

\begin{abstract}
Renal carcinoid tumors are exceedingly rare. These neuroendocrine masses are most frequently found in the gastrointestinal and respiratory tracts. A renal carcinoid tumor has only been documented in around 100 cases. In this article, we report two additional cases in female patients ages 53 and 63. Both tumors were found incidentally on computed tomography scans. Both women underwent radical nephrectomies. Neither has shown evidence of metastasis nor relapse to date; however, the 63-year-old woman was lost to followup. In conclusion, upon discovery of the asymptomatic renal mass, renal carcinoid should be a consideration in the differentiation, and if suspected, may be treated with radical nephrectomy as was done in our hospital.
\end{abstract}

Categories: Urology, Nephrology, Oncology

Keywords: primary renal carcinoid, neuroendocrine tumor, carcinoid syndrome

\section{Introduction}

Carcinoid tumors are a rare subset of neuroendocrine tumors with an incidence of 38 for every 1 million persons in the United States [1,2]. Carcinoid tumors are neuroendocrine neoplasms arising most commonly in the gastrointestinal tract and the lungs [3]. Because neuroendocrine cells are not typical of normal renal parenchyma, primary renal carcinoid tumors (PRCTs) are exceedingly rare; however, these tumors remain well-differentiated [4]. Currently, less than 100 such cases have been reported in the entire available literature [5-7]. When these tumors occur, the ability to metastasize is low. In a review of the literature, only $23 \%$ were found to metastasize, with lymph node (LN) and liver involvement being the most common locations at $18 \%$ each [8].

Review began 03/09/2021 Review ended 03/13/2021 Published 03/15/2021

() Copyright 2021 Kelly et al. This is an open access article distributed under the terms of the Creative Commons Attribution License CC-BY 4.0., which permits unrestricted use, distribution, and reproduction in any medium, provided the original author and source are credited.
The paucity of cases, indolent nature of the disease, and illusive findings on imaging complicate the diagnostic process and cause many such cases to go misdiagnosed [9]. Overall, 13\% of patients with a renal carcinoid tumor present with carcinoid syndrome [4,10]. This syndrome is a result of the tumor producing excess serotonin. When carcinoid syndrome occurs, patients present with facial flushing, wheezing, blood pressure changes (most commonly hypotension), malnutrition, and diarrhea [10]. The purpose of this report is to add to the overall knowledge base of these tumors.

\section{Case Presentation}

\section{Case 1}

A 63-year-old female with a past medical history of clinical-stage, IB-grade-2 endometrial adenocarcinoma, status post-total abdominal hysterectomy, and bilateral salpingo-oophorectomy was referred for evaluation of an incidental left renal mass found on surveillance computed tomography of the abdomen and pelvis (CT $\mathrm{A} / \mathrm{P})$.

On presentation, she denied constitutional symptoms, gross hematuria, flank pain, and lower urinary tract symptoms. The CT urogram scan of the abdomen and pelvis demonstrated a 3.8-cm heterogeneously enhancing lesion of the left lower renal pole (Figure 1). A small number of enlarged retroperitoneal LN was also identified. A two-view (posteroanterior and lateral) chest X-ray (CXR) was also obtained, noting no evidence of metastatic disease. The patient ultimately underwent a left laparoscopic retroperitoneal radical nephrectomy. 


\section{Cureus}

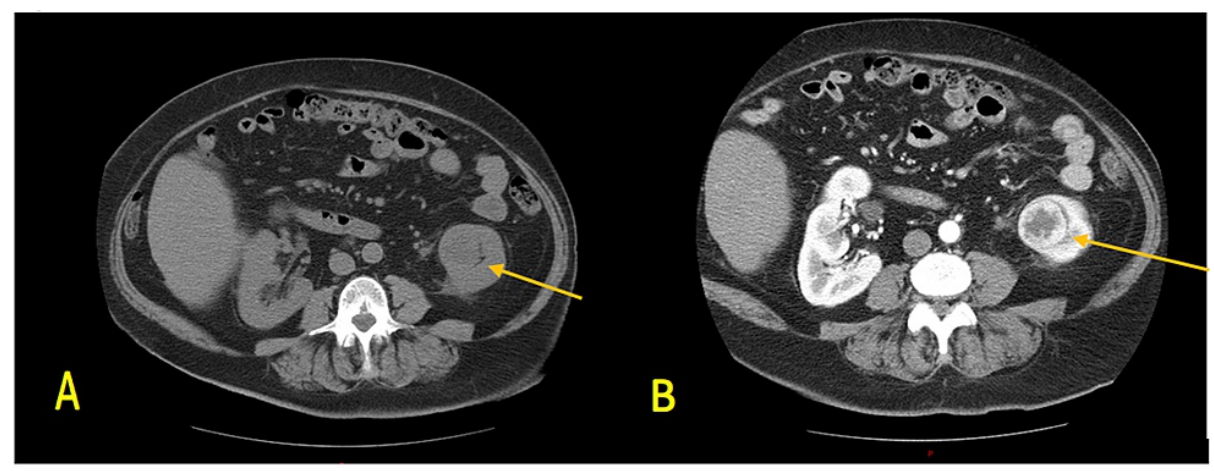

FIGURE 1: CT scan of the abdomen and pelvis without (A) and with (B) IV contrast with urogram demonstrating a $3.8-\mathrm{cm}$ heterogeneously enhancing lesion of the left lower renal pole as designated by the arrow.

$\mathrm{CT}$, computed tomography

Gross pathologic examination of the specimen revealed a $3.5 \times 3 \times 2$-cm yellow-tan mass in the lower pole involving both cortex and medulla. No renal sinus or vascular involvement was seen (Figure 2). The initial pathologic diagnosis was clear-cell renal cell carcinoma (RCC), but given the concern for metastatic endometrial cancer to the kidney, we requested that the microscopic examination be reviewed. Repeat examination with immunostaining demonstrated findings typical of a neuroendocrine malignancy with features of carcinoid tumor, arising in the background of clear-cell-type RCC. The tumor cells revealed a positive reaction for CD56 (3+) and synaptophysin (2+). The cells also contained numerous membranebound, electron-dense neuroendocrine granules (Figure 3). Microscopically, there was no angiolymphatic invasion, sinus involvement, or extracapsular extension. Final pathologic staging was pT1, N0, Mx with a histopathology grade of G2. 


\section{Cureus}

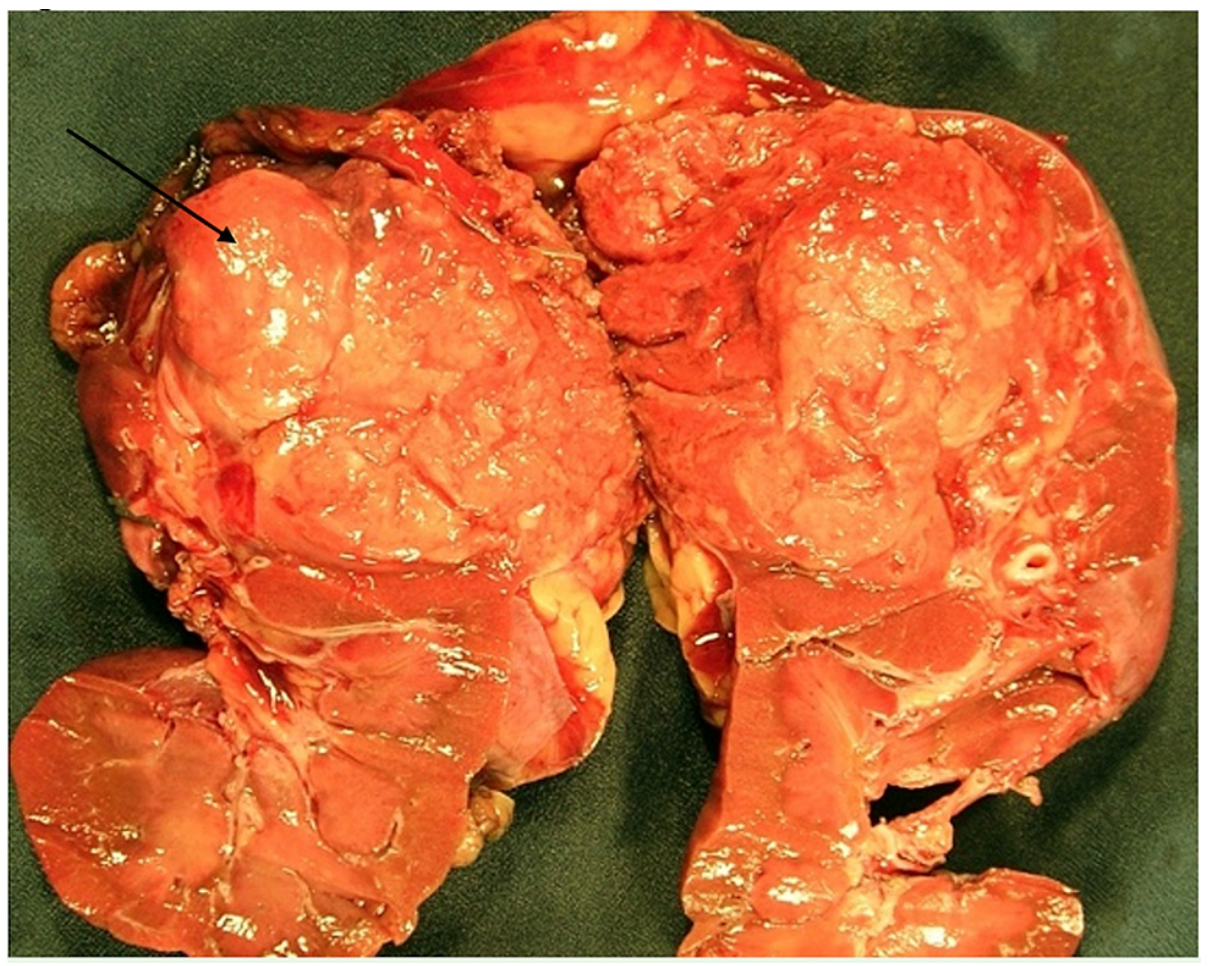

FIGURE 2: Gross kidney from radical nephrectomy. Gross pathology specimen: $3.5 \times 3 \times 2-\mathrm{cm}$ yellow-tan mass visualized in the lower pole involving both cortex and medulla as indicated by the arrow. Multiple sections revealed a soft-red, possibly necrotic, lesion in the tumor parenchyma and no renal sinus or vascular involvement. 


\section{Cureus}
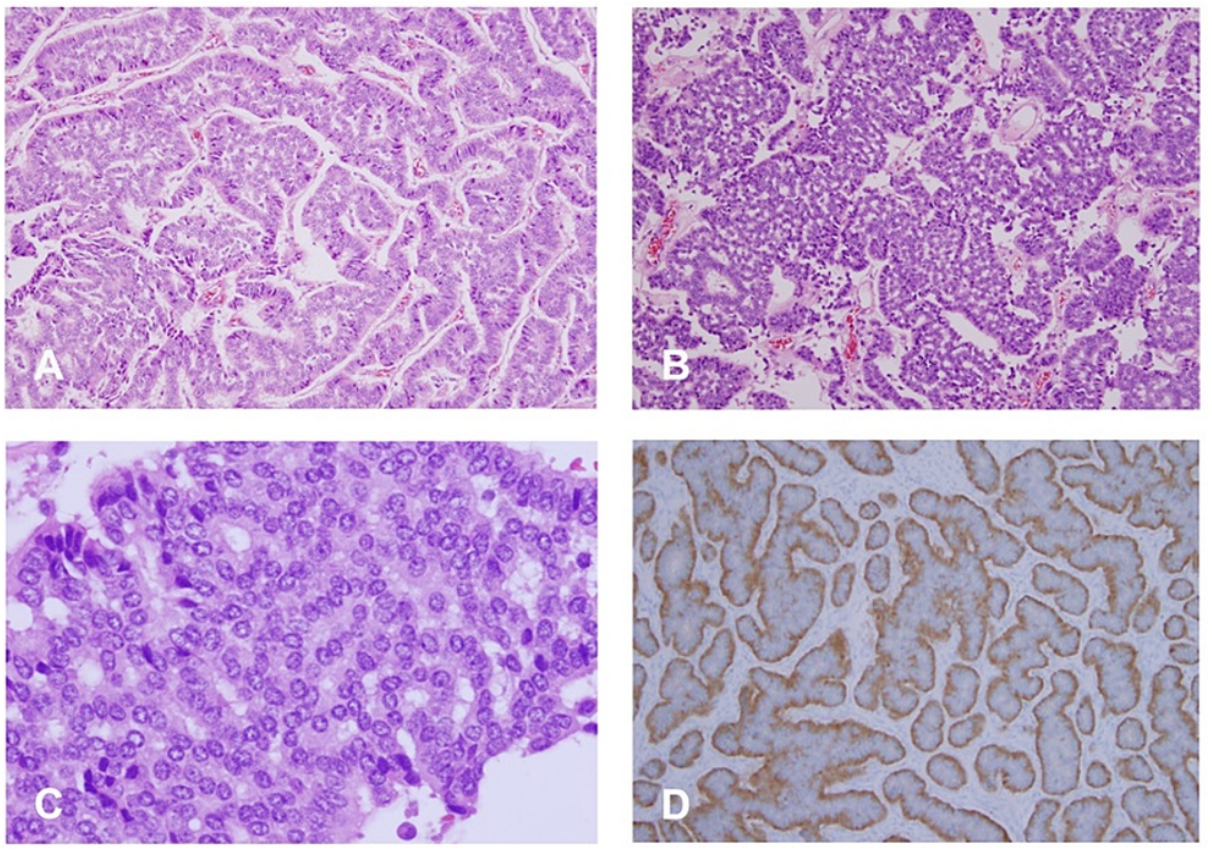

FIGURE 3: Microscopic evaluation of excised tumor. Variable growth patterns were noted. The tumor cells are arranged as trabeculae, ribbons, or nests (A, B). Solid expansile growth was also noted in focal areas. The tumor cells are uniform with limited cytoplasm and the nuclei are round. Most tumor cells showed "salt and paper" nuclear chromatin (C). Mitotic figures were rare and no geographic necrosis was seen. Immunohistochemistry revealed that the tumor cells were positive for CD56 (3+), synaptophysin (2+), and containing numerous membranebound, electron-dense neuroendocrine granules (D).

Serial surveillance CT A/P six and 12 months post-operatively demonstrated no evidence of disease with stable retroperitoneal lymphadenopathy. CT A/P at 18 months post-operatively revealed enlarging interaortocaval lymphadenopathy, measuring $2.1 \times 1.8 \mathrm{~cm}$ (compared to $2 \times 1.4 \mathrm{~cm}$ ). Following a discussion with the patient regarding observation with repeat imaging in six months versus biopsy, the patient opted for surveillance.

Over the course of the following year, the LNs continued to enlarge, with the largest measuring up to $3.7 \mathrm{~cm}$. Fine-needle aspiration of an enlarged celiac LN revealed benign hepatocytes and mixed lymphocytes. The patient was followed conservatively with a CT scan every six months for 30 months due to the benign nature of the findings. A positron emission tomography (PET)/CT scan was performed and there was mild uptake noted in the upper abdominal nodes, which was felt to be non-specific; however, low-grade lymphoma could not be excluded as there was no evidence of a metastatic carcinoid tumor. The patient was then lost to follow-up.

\section{Case 2}

A 53-year-old female was referred for evaluation of a 7 -cm right, intrapolar enhancing renal mass found incidentally on workup for right upper quadrant abdominal pain. Past medical history included hypertension, hyperlipidemia, type II diabetes, and lifelong tobacco use. The patient denied constitutional symptoms, flank pain, gross hematuria, and lower urinary tract symptoms. CT A/P demonstrated a 7-cm prominently solid but enhancing dorsal right mid renal lesion with no evidence of osseous, adrenal, or hepatic metastasis or renal venous invasion or retroperitoneal lymphadenopathy (Figure 4). A CXR was obtained, noting no evidence of metastatic disease. The patient underwent an uncomplicated right handassisted laparoscopic nephrectomy. 


\section{Cureus}

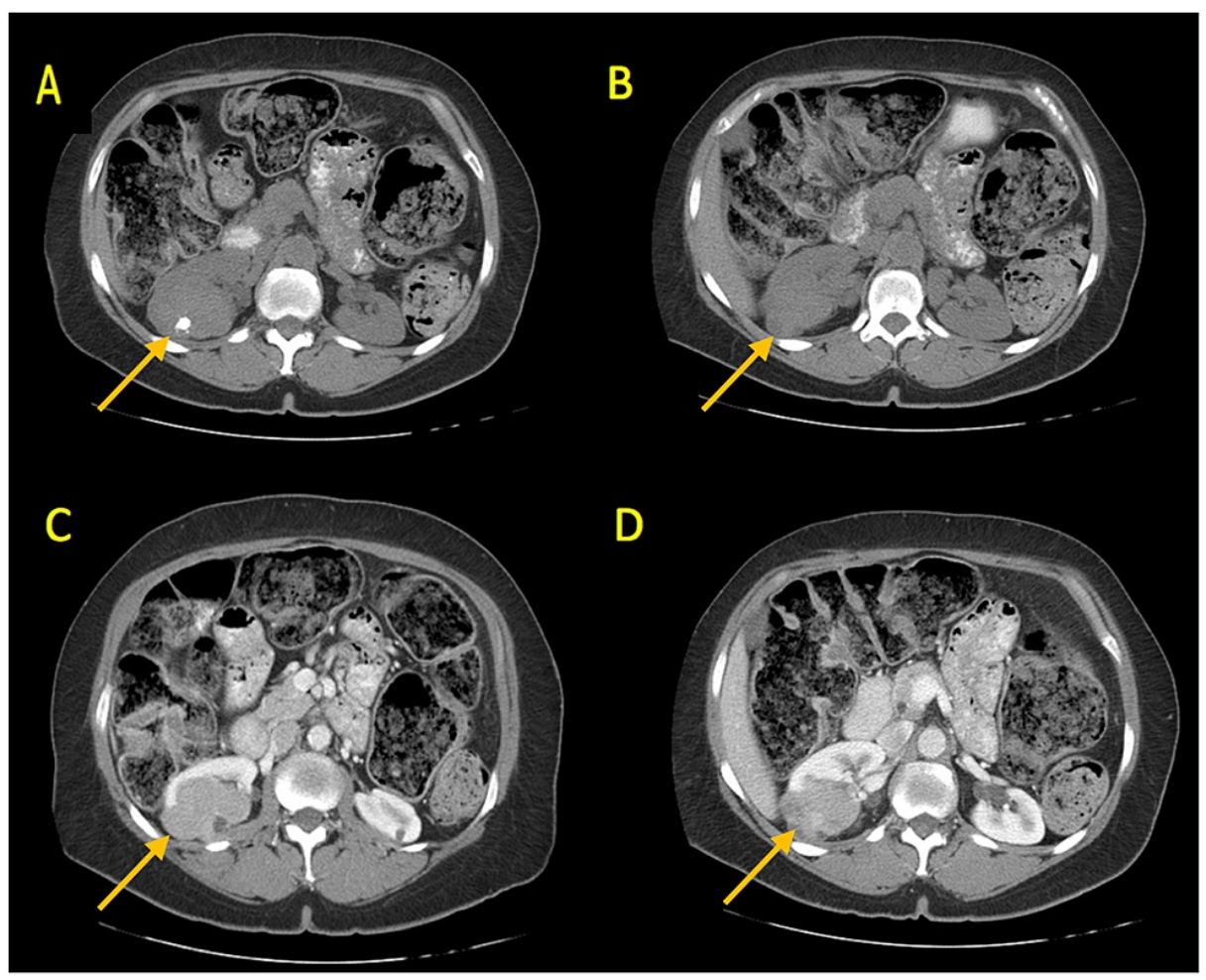

FIGURE 4: CT scan of abdomen and pelvis. Non-contrast exam demonstrating (A, B) and contrast (C, D) 7-cm prominently solid but enhancing dorsal right mid renal lesion with no evidence of osseous, adrenal, or hepatic metastasis or renal venous invasion or retroperitoneal lymphadenopathy as demonstrated by the arrow.

Gross pathology revealed a well-demarcated tan solid tumor of the upper pole measuring $6.0 \times 6.5 \times 3 \mathrm{~cm}$. The tumor appeared to reveal variable parenchyma morphological expression in both growth patterns and cytomorphology. Immunohistochemical stains were positive for CD56 and synaptophysin with membranebound, electron-dense neuroendocrine granules consistent with primary renal carcinoid (Figure 5). Final pathologic staging was pT1, Nx, Mx with a histopathology grade of G2. 


\section{Cureus}
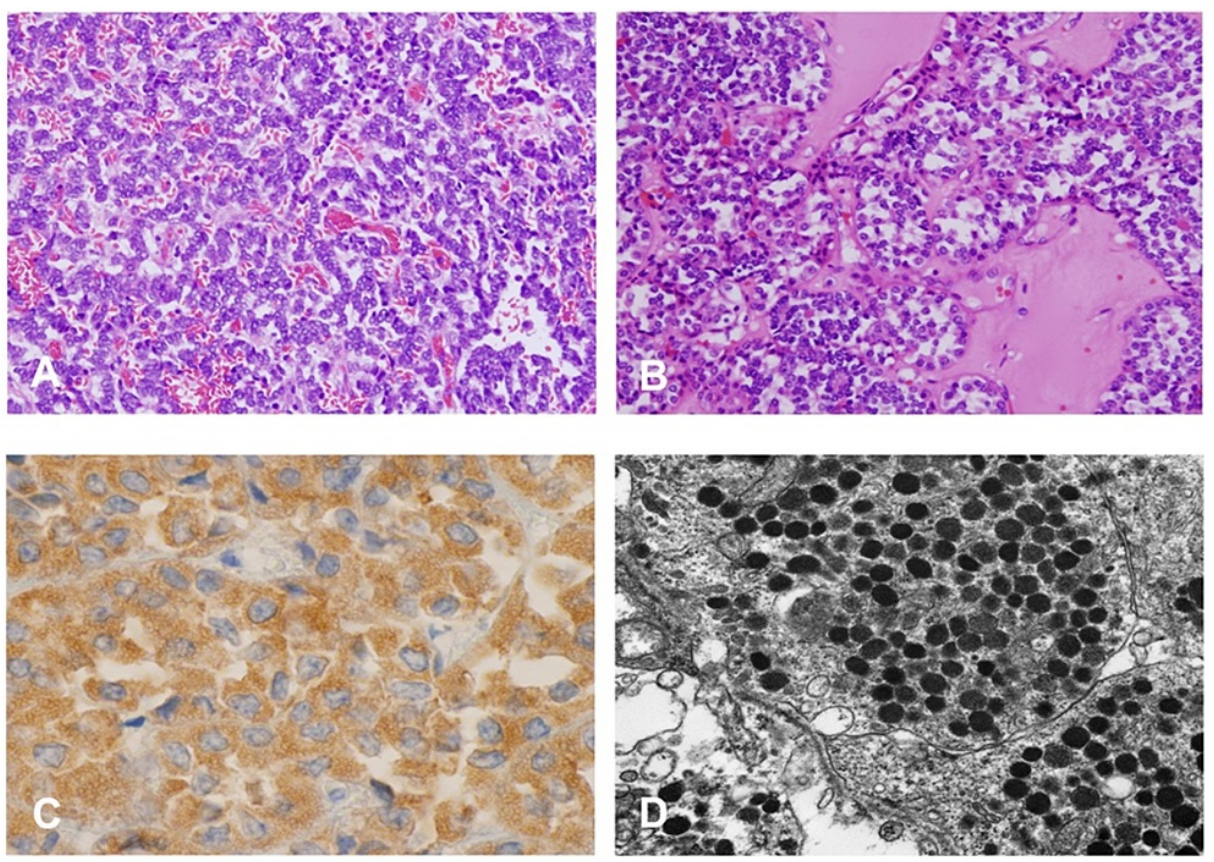

FIGURE 5: Pathological analysis of the resected tumor. Microscopic evaluation: solid, nest, and sheeting growth of tumor cells were presented in a hyalinized collagenous stroma. The tumor cells were uniform and contain eosinophilic or optically clear cytoplasm with rare mitoses. No angiolymphatic invasion or sinus involvement was identified (A, B). The tumor cells showed a positive reaction for CD56 and synaptophysin in the immunohistochemical study (C) and membrane-bound, electron-dense neuroendocrine granules in ultrastructural examination (D).

Four months after the removal of the tumor, serotonin level and urine 5-HIAA were within normal range and a surveillance octreotide scan revealed a suspicious focus of abnormal activity in the right abdomen concerning for primary carcinoid tumor versus metastasis (Figure ๑). An upper GI endoscopy revealed a number of gastric ulcers positive for Helicobacter pylori. PET scan was unremarkable. CT chest, A/P, performed six months post-operatively showed no evidence of recurrence and resolution of the abdominal mass. We continued with yearly surveillance CT. Serotonin and metabolite levels were obtained twice yearly. The patient remained without recurrence until the last documented follow-up. 

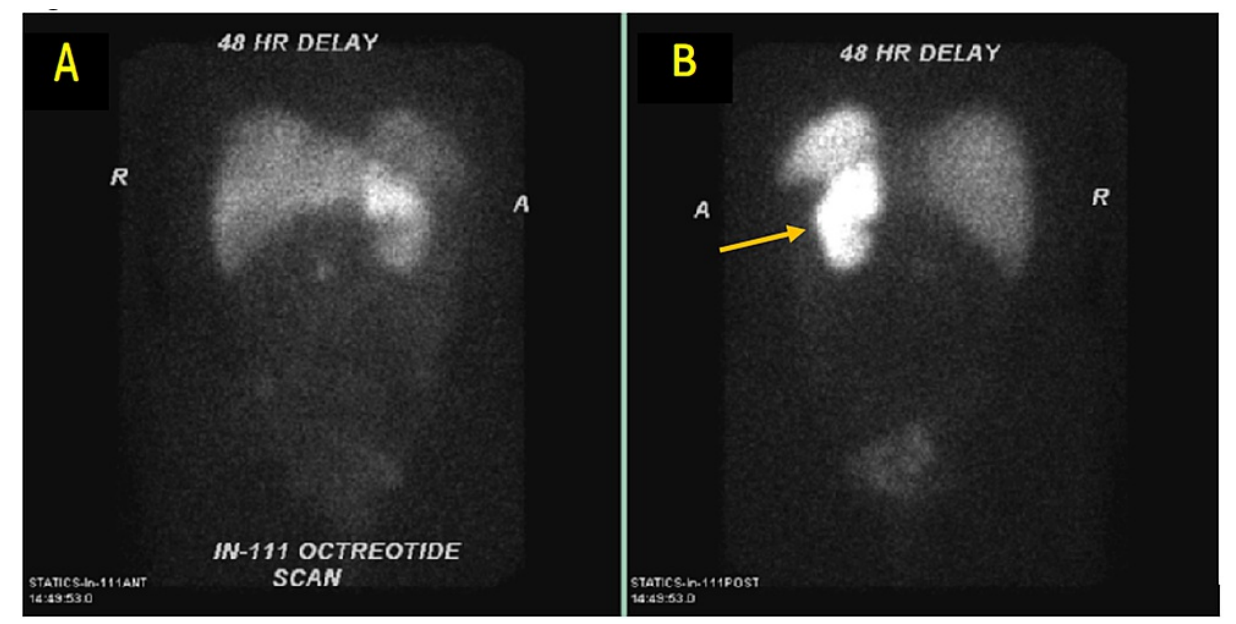

FIGURE 6: Octreotide uptake scan. Octreotide scan (360-degree view): suspicious focus of abnormal activity in the right abdomen, likely the third portion of the duodenum, which may represent primary carcinoid tumor versus a metastatic lesion. Please note right kidney is surgically absent and the area of the left kidney enhances as octreotide is absorbed normally by native kidney cells.

\section{Discussion}

PRCTs are very rare neoplasms of neuroendocrine differentiation arising within the renal parenchyma. Patients are often asymptomatic at presentation, but when symptomatic, may have localized or systemic symptoms. Approximately 25-30\% are incidental findings, though patients can present with abdominal pain, flank pain, hematuria, constipation, constitutional symptoms, and carcinoid syndromes. These malignancies are more often associated with preexisting renal pathologies, including horseshoe kidney, teratomas, and polycystic kidney disease [9]. There are also reports of these malignancies arising within another primary renal malignancy, including another report of a primary renal carcinoid tumor arising in a background of clear-cell-type RCC [11].

Although radiographic features do not consistently differentiate these neoplasms from other renal masses, CT and octreotide scintigraphy are routinely used to aid in diagnosis and post-operative surveillance of disease [12]. Because of the non-specific clinical and radiologic presentation of these tumors, histology and immunohistology staining is necessary for diagnosis. Uniform collection of ovoid cells forming a trabecular mass with a pseudopapillary structure is the characteristic histologic appearance. Synaptophysin, CgA, S100, CD56, neuron-specific enolase, serotonin, and VIP are common positive markers of neuroendocrine differentiation. Therefore, primary renal carcinoids characteristically stain positive for one or more of the aforementioned markers and negative for urothelial and renal cell markers (CK7, CK20, PAX-2, PAX-8, and CD10) [12-16].

Incidental finding in case 1 and presentation with abdominal pain of another patient in fifth to sixth decade of age is similar to reported cases. Both patients underwent CT A/P, each of which demonstrated a unilateral enhancing renal mass. Subsequent CXRs ruled out metastatic disease in both cases. They both ultimately underwent laparoscopic unilateral radical nephrectomy. Immunohistochemical staining demonstrated positive staining for CD56 and synaptophysin in both cases. Surveillance during the post-operative period differed between patients, despite having the same primary diagnosis and receiving care at the same institution. The non-specific presentation, non-discriminatory pre-operative imaging, surgical treatment, characteristic neuroendocrine pathology, and variable surveillance methods in these cases closely mimic the majority of other rare cases reported in the current literature.

Despite these shared characteristics, some major variability in case presentation still exists and may account for current (and historical) challenges in diagnosis and management. At the time of presentation, case 1 was asymptomatic with an incidental enhancing mass on the lower pole of the left kidney, which was subsequently misdiagnosed as RCC. Upon further pathological workup, a diagnosis of PRCT within the background of clear-cell-type RCC was made. Surveillance included six-month CT A/P for 18 months until the patient was lost to follow-up. Alternatively, case 2 presented with right upper quadrant abdominal pain and an enhancing mass on the upper pole of the right kidney measuring nearly twice the size of the first patient's mass. However, the course of surveillance for this case was more complex. Surveillance for this patient included a four-month post-operative measurement of serum serotonin and urine 5-HIAA in 
addition to an octreotide scan with subsequent endoscopy and PET scan. Further testing at six months postoperatively included a CT of the chest and A/P, which was repeated annually thereafter. Measurements of serum markers and urine metabolites were also repeated biannually. Neither patient showed evidence of metastatic disease throughout the surveillance period, although one patient was eventually lost to followup. These outcomes are consistent with the well-documented favorable prognosis that follows complete resection of PRCT.

\section{Conclusions}

This case report highlights the variability in presentation leading to difficulty in the diagnosis of renal carcinoid tumors. It is crucial to differentiate this tumor from other renal masses. If a lesion is found within RCC, the management of the patient must be adjusted accordingly. Given the rarity of the condition, there is a lack of follow-up protocols and treatment plans. More research is needed for renal carcinoid tumors.

\section{Additional Information \\ Disclosures}

Human subjects: Consent was obtained or waived by all participants in this study. Institutional Review Board issued approval STUDY00001015. The IRB determined that the proposed activity is not research involving human subjects as defined by DHHS and FDA regulations. Further IRB review and approval by this organization is not required. This determination applies only to the activities described in this IRB submission and does not apply should any changes be made. If changes are made you must immediately contact the IRB about whether these activities are research involving humans in which the organization is engaged. You will also be required to submit a new request to the IRB for a determination. If you have any questions, please contact the IRB Administrative Office at (318) 813-1350. Sincerely, IRB Administration. Conflicts of interest: In compliance with the ICMJE uniform disclosure form, all authors declare the following: Payment/services info: All authors have declared that no financial support was received from any organization for the submitted work. Financial relationships: All authors have declared that they have no financial relationships at present or within the previous three years with any organizations that might have an interest in the submitted work. Other relationships: All authors have declared that there are no other relationships or activities that could appear to have influenced the submitted work.

\section{References}

1. Maggard MA, O'Connell JB, Ko CY: Updated population-based review of carcinoid tumors. Ann Surg. 2004, 240:117-122. 10.1097/01.sla.0000129342.67174.67

2. Modlin IM, Lye KD, Kidd M: A 5-decade analysis of 13,715 carcinoid tumors . Cancer. 2003, 97:934-959. 10.1002/cncr.11105

3. Chen CT, Hsieh SW, Hsieh TS: Case report: a case of primary renal carcinoid tumor . Urol Case Rep. 2018, 21:14-16.10.1016/j.eucr.2018.07.020

4. Jabaji R, Kern T, Shen D, Chu W, Merchant M: Primary renal carcinoid tumor: report of two cases. Perm J. 2020, 24:147. 10.7812/tpp/19.147

5. Linke CS, Shie S: An asymptomatic primary renal carcinoid tumor: a case report . Urol Case Rep. 2016, 7:7071.10.1016/j.eucr.2016.04.018

6. Tanaka T, Yamamoto H, Imai A, et al.: A case of primary renal carcinoid tumor . Case Rep Urol. 2015, 2015:736213. 10.1155/2015/736213

7. Ouyang B, Ma X, Yan H, He J, Xia C, Yu H: Renal carcinoid tumor with liver metastasis followed up postoperatively for 9 years. Diagn Pathol. 2015, 10:182. 10.1186/s13000-015-0417-7

8. Shurtleff BT, Shvarts O, Rajfer J: Carcinoid tumor of the kidney: case report and review of the literature. Rev Urol. 2005, 7:229-233.

9. Romero FR, Rais-Bahrami S, Permpongkosol S, Fine SW, Kohanim S, Jarrett TW: Primary carcinoid tumors of the kidney. J Urol. 2006, 176:2359-2366. 10.1016/j.juro.2006.07.129

10. Rosenberg JE, Albersheim JA, Sathianathen NJ, Murugan P, Weight CJ: Five new cases of primary renal carcinoid tumor: case reports and literature review. Pathol Oncol Res. 2020, 26:341-346. 10.1007/s12253018-0481-x

11. Bressenot A, Delaunay C, Gauchotte G, Oliver A, Boudrant G, Montagne K: Composite renal cell carcinoma with clear cell renal cell carcinomatous and carcinoid tumoral elements: a first case report. J Clin Pathol. 2010, 63:174-176. 10.1136/jcp.2009.070292

12. Lamb L, Shaban W: Primary renal carcinoid tumor: a radiologic review . Radiol Case Rep. 2014, 9:923. 10.2484/rcr.v9i2.923

13. Daboul N, Monga D, Bunker M: Primary renal carcinoid tumour with lung metastasis misdiagnosed as renal cell carcinoma. BMJ Case Rep. 2016, 2016:2015213432. 10.1136/bcr-2015-213432

14. McCaffrey JA, Reuter VV, Herr HW, Macapinlac HA, Russo P, Motzer RJ: Carcinoid tumor of the kidney. The use of somatostatin receptor scintigraphy in diagnosis and management. Urol Oncol. 2000, 5:108-111. 10.1016/S1078-1439(99)00050-2

15. Shi W, Johnston CF, Buchanan KD, Ferguson WR, Laird JD, Crothers JG, McIlrath EM: Localization of neuroendocrine tumours with [111In] DTPA-octreotide scintigraphy (Octreoscan): a comparative study with CT and MR imaging. QJM. 1998, 91:295-301. 10.1093/qjmed/91.4.295

16. Kirshbom PM, Kherani AR, Onaitis MW, Feldman JM, Tyler DS: Carcinoids of unknown origin: comparative analysis with foregut, midgut, and hindgut carcinoids. Surgery. 1998, 124:1063-1070.

10.1067/msy. 1998.93105 